\title{
ANTIRETROVIRAL THERAPY AND BONE HEALTH
}

\author{
N. Yancheva ${ }^{1}$, N. Temelkova² D. Strashimirov' ${ }^{1}$ I. Gabarska ${ }^{1}$, T. Tchervenyakova ${ }^{1}$ \\ 1Department of AIDS, "Professor Ivan Kirov" Specialized Hospital for Infectious and Parasitic Diseases, \\ Medical University - Sofia, Bulgaria \\ ${ }^{2}$ Department of Clinical Densitometry and Metabolic Disorders, Aleksandrovska University Hospital - Sofia, Bulgaria
}

\begin{abstract}
Objective: To determine the incidence of osteopenia and osteoporosis in Bulgarian human immunodeficiency virus (HIV)-infected patients. Methods: Bone mineral density (BMD) was measured by dual-energy X-ray absorptiometry in 41 Bulgarian HIV-infected patients. Results: Overall, $27 \%$ of patients had reduced BMD (7\% osteoporosis and $20 \%$ osteopenia). There was a significant correlation between low BMD and increasing age and between low BMD and duration of antiretroviral therapy (ART) but no correlation with gender, therapy regimen or serum calcium or phosphate levels. Seventy-one percent had elevated serum cystatin $C$ levels but there was no correlation between BMD and cystatin $C$ levels. Serum calcium, phosphate, cystatin $C$ and creatinine levels and the $T$ - and Z-scores were tested for dependence on duration of therapy. Dependence was found for $T$ - and Zscores, ( $p=0.048$ and $p=0.038$ ) but not for calcium, phosphate, cystatin $C$ or creatinine levels. These variables were subsequently tested by means of cross-tabulation tables for correlations between pairs of parameters; no significant correlation was found between any pairs ( $R>0.5$ for all comparisons). Conclusions: Significant correlations were observed between low BMD and increasing age and longer duration of ART but no relationship was observed with gender or therapy regimen.
\end{abstract}

Key words: HIV, antiretroviral therapy, bone mineral density, renal function

Corresponding author: Nina Yancheva-Petrova, MD, PhD, Chief of Department of HIVIAIDS, "Prof. Ivan Kirov" Specialized Hospital for Infectious and Parasitic Diseases, Assistant Professor, Department of Infectious Diseases, Parasitology and Tropical Medicine, Medical University, 17 Acad. Ivan Geshov Blvd, 1431 Sofia, Bulgaria, tel.: +35 98785 0904, e-mail dr.yancheva@abv.bg

\section{INTRODUCTION}

L ow bone mineral density (BMD) is prevalent in human immunodeficiency virus (HIV)-infected subjects and the increased risk of osteoporosis and fractures associated with HIV infection, and some antiretroviral agents, is well established [16, 28].

Osteoporosis is a common skeletal disorder which increases the risk of fractures [2, 21, 30]. It is characterized by impaired bone strength, which is de- termined by both bone density and bone quality. Primary osteoporosis is usually associated with postmenopausal oestrogen loss (osteoporosis type 1 ) or age-related deterioration of bone architecture (osteoporosis type 2); idiopathic osteoporosis in young people is rare [29]. Secondary osteoporosis may be due to endocrine disorders, such as hypercorticism, hypogonadism and hyperthyroidism; malabsorption syndrome leading to calcium deficiency; severe immobility resulting in disturbed bone metabolism [18, 
30]. Prolonged glucocorticoid treatment is the most common cause of iatrogenic osteoporosis. Some congenital diseases such as osteogenesis imperfecta are also associated with osteoporosis [22, 33]. Osteoporosis is usually generalised but localised forms can also occur [20].

Osteoporosis is characterised by a decrease in BMD, which can be quantified as a T-score or a Zscore. The T-score is the number of standard deviations (SDs) above or below the mean BMD for a healthy 25-29-year-old adult of the same sex and ethnicity [19], while the Z-score is the number of SDs above or below the mean for the patient's age, sex and ethnicity. According to the World Health Organization (WHO) classification, a T-score of -1.0 or higher indicates a normal BMD, while osteopenia is defined as a T-score between -1.0 and -2.5 and osteoporosis is a T-score of -2.5 or lower [37]. Severe osteoporosis is defined as a T-score $\leq-2.5$ SDs, with a fracture.

Data from a number of cross-sectional studies involving both young and elderly HIV-infected patients showed a high incidence of reduced BMD [3, 4, 16, $24,26]$. According to one study, osteoporosis is three times more common among HIV-infected patients compared with HIV-negative controls, especially in patients receiving anti-retroviral therapy (ART) [3]. ART has been associated with a $2-6 \%$ decrease in BMD during the first two years of treatment, which is similar to the decrease in BMD that occurs during the first two years of the menopause [25]. Other studies show a higher incidence of fractures in HIV-infected patients compared with HIV-negative controls (30$70 \%$ increase in risk) [3].

The causes of low BMD in HIV-infected patients are multifactorial and constitute a complex of interactions between the HIV infection itself, traditional osteoporosis risk factors (poor diet and low weight, smoking, alcohol use and low vitamin D levels) and ART [3, $4,24,26,38]$. Osteopenia is also commonly found in ART-naive patients, indicating that uncontrolled viraemia may affect BMD, possibly mediated by the effect of systemic inflammation on bone remodelling [26]. HIV proteins increase the resorptive activity of osteoclasts and decrease bone formation by promoting osteoblast apoptosis [25]. In addition, the increased levels of tumour necrosis factor induce increased osteoclast-mediated bone resorption without the associated elevation of bone formation [25].

Co-morbid conditions in patients with HIV infection also affect BMD. Serum vitamin D levels are frequently low in HIV-infected patients [34, 38, 39, 40]. According to many international studies, $60-75 \%$ of HIV-infected patients have lower vitamin D levels than healthy individuals without HIV infection [34]. This percentage is even higher in the Bulgarian population, at 83\% [38]. Other causes of low BMD in HIV-infected patients include hypogonadism and lipoatrophy [4, 25]. The onset of the ART sometimes induces a statistically and clinically significant loss of BMD, regardless of the initial treatment regimen [3] and some studies indicate that the risk may be higher with regimens containing the nucleoside reverse transcriptase inhibitor, tenofovir disoproxil fumarate (TDF) [14, 28].

Renal tubular toxicity has also been reported with ART, including the TDF-containing regimens [7, 10, 15] and may increase the risk of bone loss; the risk of nephrotoxicity is increased in patients with pre-existing chronic kidney disease, an important comorbid condition in the HIV-positive population $[1,7,10,11$, $15,36]$. Consequently, we also evaluated factors related to kidney function, including the measurement of serum cystatin C levels. Cystatin $C$ is a $13 \mathrm{kDa}$ protein which is freely filtered across the glomeruli, reabsorbed and fully catabolised by the proximal renal tubules $[1,8]$. It is a more reliable and specific marker of renal function, particularly of early renal impairment, than serum creatinine, since its production is constant and its concentration is dependent only on the glomerular filtration rate (GFR) and is not influenced by sex, age, race, protein intake or muscle mass [8, 17, 31]. Serum cystatin $C$ levels may therefore be more sensitive to a decrease in GFR than serum creatinine levels.

The aim of our study was to analyse BMD in HIVinfected Bulgarian patients. We measured BMD at the spine and hip by dual-energy X-ray absorptiometry (DEXA) scanning, which is rapid, accurate, and sensitive and involves very low radiation exposure [2, 23]. As mentioned above, we also evaluated factors related to kidney function.

\section{MATERIAL AND METHODS}

BMD was measured in $41 \mathrm{HIV}$-infected patients treated at the Department of Acquired Immune Deficiency at the Professor Ivan Kirov Specialized Hospital for Infectious and Parasitic Diseases, Sofia. DEXA scans were performed at the Department of Clinical Densitometry and Bone Metabolic Diseases of Aleksandrovska University Hospital, Sofia.

In addition to the routine laboratory tests that were performed during HIV monitoring, serum cystatin C, calcium and phosphate levels were measured. The laboratory tests were carried out in the clinical laboratory of the Professor Ivan Kirov Specialized Hospital for Infectious and Parasitic Diseases. 
This work was carried out in accordance with The Code of Ethics of the World Medical Association (Declaration of Helsinki) for experiments involving humans; informed consent was obtained from all participants prior to enrolment.

\section{STATISTICAL ANALYSIS}

The results were analysed statistically using the descriptive analysis methods of the frequency of the distribution of the studied parameters (variables), evaluation of the correlation between the variables by determining the correlation coefficient, $\chi^{2}$ test and Friedman's two-factor analysis of variance.

\section{RESULTS}

Laboratory and BMD measurements were performed on 41 patients, 32 men and 9 women. The patient characteristics are shown in Table 1.

All patients included in this study received ART; in total there were 15 different antiretroviral regimens, summarised in Table 2. The mean duration of ART was $46.8 \pm 46.5$ months, with a range of 7-204 months.

In most patients $(30 ; 73 \%)$, the BMD was in the normal range. In total, $27 \%$ of patients had reduced BMD, of which $3(7 \%)$ had osteoporosis and $8(20 \%)$ had osteopenia. The distribution of patients according to BMD is presented in Table 3.

Table 1. Patient characteristics

\begin{tabular}{|c|c|c|c|}
\hline & $\begin{array}{l}\text { All patients } \\
(\mathrm{n}=41)\end{array}$ & $\begin{array}{l}\text { TDF-based regimen } \\
(n=24)\end{array}$ & $\begin{array}{l}\text { Non-TDF-based regimen } \\
(n=17)\end{array}$ \\
\hline Mean age, years & 38.7 & 37.8 & 40.1 \\
\hline Gender male/female, $\mathrm{n}$ & $32 / 9$ & $18 / 6$ & $14 / 3$ \\
\hline Mean CD4 count at start of treatment per $\mathrm{mm}^{3}$ & $305 \pm 101$ & $305 \pm 101$ & $297 \pm 170$ \\
\hline Mean viral load at start, copies $/ \mathrm{ml}$ & $67,364 \pm 51,351$ & $66,228 \pm 51,616$ & $68,967 \pm 52,513$ \\
\hline Mean duration of ART, months & $46.8 \pm 46.5$ & $36.6 \pm 39.0$ & $61.2 \pm 61.2$ \\
\hline Mean serum creatinine, $\mathrm{mmol} / \mathrm{l}$ & $76.46 \pm 14.89$ & $79.2 \pm 17,7$ & $72.5 \pm 8,6$ \\
\hline Mean eGFR, $\mathrm{ml} / \mathrm{min}$ & $108 \pm 26,4$ & $106.1 \pm 31,1$ & $110.5 \pm 18.5$ \\
\hline Mean cystatin C, mg/l & $1.21 \pm 0.39$ & $1.29 \pm 0.34$ & $1.12 \pm 0.43$ \\
\hline $\begin{array}{l}\text { Mean serum phosphate, } \mathrm{n}(\%) \\
>1.45 \mathrm{mmol} / \mathrm{l} \\
0.87-1.45 \mathrm{mmol} / \mathrm{l} \\
<0.87 \mathrm{mmol} / \mathrm{l}\end{array}$ & \begin{tabular}{|l|}
$5(12.2)$ \\
$30(73.2)$ \\
$6(14.6)$ \\
\end{tabular} & 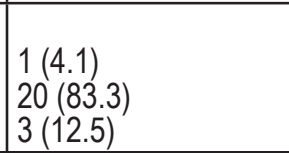 & \begin{tabular}{|l|}
$4(23.5)$ \\
$10(58.8)$ \\
$3(17.6)$ \\
\end{tabular} \\
\hline $\begin{array}{l}\text { Mean serum calcium, } \mathrm{n}(\%) \\
>2.6 \mathrm{mmol} / \mathrm{l} \\
2.2-2.6 \mathrm{mmol} / \mathrm{l}\end{array}$ & $\begin{array}{l}23(56.1) \\
18(43.9) \\
\end{array}$ & $\begin{array}{l}14(58.3) \\
10(41.7) \\
\end{array}$ & \begin{tabular}{|l}
$9(52.9)$ \\
$8(47.1)$ \\
\end{tabular} \\
\hline
\end{tabular}

Abbr.: ART, antiretroviral therapy; eGFR, estimated glomerular filtration rate; TDF, tenofovir disoproxil fumarate

Table 2. Antiretroviral treatment regimens

\begin{tabular}{|l|c|c|}
\hline Regimen & Number of patients & $\%$ \\
\hline TDF/FTC+EFV & 2 & 4.9 \\
\hline $3 T C / A B C+R A L$ & 1 & 2.4 \\
\hline TDF/FTC+ATZ/r & 1 & 2.4 \\
\hline TDF/FTC+RAL & 4 & 9.8 \\
\hline TDF+AZT+LPV/r & 1 & 2.4 \\
\hline $3 T C / A B C+E F V$ & 3 & 7.3 \\
\hline TDF/FTC+LPV/r & 4 & 9.8 \\
\hline $3 T C / A B C+D R V / r$ & 6 & 14.6 \\
\hline TDF/FTC+DRV/r & 10 & 24.4 \\
\hline TDF/FTC+DTV & 2 & 4.9 \\
\hline DRV/r+RAL & 1 & 2.4 \\
\hline $3 T C / A B C+R P V$ & 2 & 4.9 \\
\hline $3 T C / A B C+L P V / r$ & 2 & 4.9 \\
\hline $3 T C / A Z T+A T Z / r$ & 1 & 2.4 \\
\hline $3 T C / A B C+A T Z / r$ & 1 & 2.4 \\
\hline
\end{tabular}

Abbr.: 3TC, lamivudine; ABC, abacavir; ATZ/r, atazanavir/ritonavir; AZT, zidovudine; DRV/r, darunavir/ritonavir; DTV, dolutegravir; EFV, efavirenz; FTC, emtricitabine; LPV/r, lopinavir/ritonavir; RAL, raltegravir; RPV, rilpivirine; TDF, tenofovir disoproxil fumarate 
Table 3. Patient characteristics according to BMD

\begin{tabular}{|l|c|c|c|}
\hline & Normal BMD $(\mathbf{n}=\mathbf{3 0})$ & Osteoporosis $(\mathbf{n = 3})$ & Osteopenia $(\mathbf{n}=\mathbf{8})$ \\
\hline Mean T-score & -0.68 & -2.73 & -1.89 \\
\hline Mean Z-score & -0.53 & -2.53 & -1.46 \\
\hline Serum creatinine, $\mathrm{mmol} / \mathrm{l}$ & 76.39 & 78.63 & 75.90 \\
\hline eGFR, $\mathrm{ml} / \mathrm{min}$ & 113.63 & 97.67 & 90.75 \\
\hline Cystatin C, $\mathrm{mg} / \mathrm{l}$ & 1.18 & 1.29 & 1.32 \\
\hline Serum phosphate, $\mathrm{mmol} / \mathrm{l}$ & 1.15 & 1.12 & 1.06 \\
\hline Serum calcium, $\mathrm{mmol} / \mathrm{l}$ & 2.47 & 2.44 & 2.37 \\
\hline
\end{tabular}

Abbr.: BMD, bone mineral density; eGFR, estimated glomerular filtration rate

Table 4 shows the age, ART regimen and duration of therapy in patients with osteopenia or osteoporosis. Eight of the patients with reduced BMD had been treated with a TDF-containing regimen; eight had received a protease inhibitor (PI)-based ART regimen, which was lopinavir/ritonavir (LPV/r) in five cases. Three patients with osteopenia had received an ART regimen based on the non-nucleoside reverse transcriptase inhibitor, efavirenz (EFV).

Most patients $(n=29,71 \%)$ had elevated levels of serum cystatin C (upper limit of normal $0.96 \mathrm{mg} / \mathrm{l}$ ); the mean level was $1.16 \pm 1.09 \mathrm{mg} / \mathrm{l}$, with a range of 1.0 $2.25 \mathrm{mg} / \mathrm{l}$. Serum creatinine and alkaline phosphatase levels were in the normal range in all patients in this study. The mean serum creatinine level was $76.46 \pm 14.89 \mu \mathrm{mol} / \mathrm{l}$, with a range of 42.30-122.90 $\mu \mathrm{mol} / \mathrm{l}$. Serum calcium levels were above normal (> $2.6 \mathrm{mmol} / \mathrm{l})$ in $44 \%$ of patients; serum phosphate levels were above normal in $15 \%$ and below normal in $12 \%$ of patients (Table 1 ).

In order to evaluate a possible association between BMD and age, patients were divided in three age groups as follows: $<40,40-50$ and $>50$ years; a positive correlation was observed between increasing age and BMD ( $p=0.017)$. However, no correlation was found between BMD and the therapeutic regimen $(p=0.076)$. No statistically significant relationships were observed between BMD and gender $(p=$ $0.557), B M D$ and serum calcium levels $(p=0.688)$, BMD and serum phosphate $(p=0.858)$ or BMD and cystatin $C$ levels $(p=0.980)$.

We tried to analyse the allocations of these parameters using Friedman's two-factor analysis of variance, with the null hypothesis that the parameters tested were of identical distribution. The null hypothesis was rejected ( $p=0.0001)$, i.e. they were not identical. A statistically significant relationship between the indicators was not detected $(p=0.200)$.

Serum calcium, phosphate, cystatin $C$ and creatinine levels and the $\mathrm{T}$ - and Z-scores were tested for dependence on the duration of therapy. For T- and Z-scores dependence was found ( $p=0.048$ and $p=0.038$ ). No dependence was found for calcium $(p=0.735)$, phosphate $(p=0.083)$, cystatin C $(p=0.244)$ or cre-

Table 4. Details of ART therapy in patients with decreased BMD

\begin{tabular}{|l|l|l|l|l|l|l|}
\hline & \multicolumn{3}{|c|}{ Osteoporosis $(\mathbf{n}=\mathbf{3})$} & \multicolumn{3}{c|}{ Osteopenia $(\mathbf{n}=8)$} \\
\hline & Age, years & ART regimen & Duration, months & Age, years & ART regimen & Duration, months \\
\hline Male & 33 & 3TC/ABC + EFV & 93 & 40 & 3TC/ABC + ATZ/r & 17 \\
& 45 & TDF/FTC + EFV & 26 & 43 & TDF/FTC + DRV & 23 \\
& 54 & TDF/FTC + LPV/r & 46 & 61 & $3 T C / A B C+L P V / r$ & 42 \\
& & & & 46 & TDF/FTC + LPV/r & 130 \\
\hline Female & & & & 40 & TDF/FTC + LPV/r & 58 \\
& & & & 55 & TDF/FTC + EFV & 63 \\
& & & 44 & TDF + LPV/r & 102 \\
& & & 39 & TDF/FTC + LPV/r & 130 \\
\hline
\end{tabular}

Abbr.: 3TC, lamivudine; ABC, abacavir; ART, antiretroviral therapy; ATZ/r, atazanavir/ritonavir; BMD, bone mineral density; DRV, darunavir; EFV, efavirenz; FTC, emtricitabine; LPV/r, lopinavir/ritonavir; TDF, tenofovir disoproxil fumarate 
atinine levels $(p=0.277)$. For BMD, serum calcium and cystatin $C$ there is a $99.9 \%$ probability of abnormalities. The serum calcium, phosphate, cystatin $\mathrm{C}$ and creatinine levels and the $\mathrm{T}$ - and Z-scores were subsequently tested by means of cross-tabulation tables for correlations between any pair of parameters - calcium and T-score, calcium and Z-score, calcium and phosphate, phosphate and T-score, phosphate and Z-score and T- and Z-score. No significant correlation was found between any pair of parameters ( $R>0.5$ for all comparisons).

\section{DISCUSSION}

HIV-infected patients are at risk for the development of osteopenia and osteoporosis as a result of many factors, including HIV infection itself, immune suppression, life-long ART, vitamin D deficiency and hypogonadism [3, 4, 12, 26, 27, 28, 38]. To the best of our knowledge, this study is the first investigation of the incidence of osteopenia and osteoporosis to be undertaken in a Bulgarian HIV population. A relatively small number of patients agreed to have their BMD measured; this reluctance was probably due to the need to attend a different clinic where their diagnosis would have to be revealed. Nevertheless, these preliminary results show that osteopenia or osteoporosis were present in $27 \%$ of HIV-infected patients enrolled in the study.

There was a significant correlation between reduced BMD and increasing age, but no correlation with gender or therapy regimen. However, there was a statistically significant relationship between low BMD and the duration of ART. Another interesting result of our study is the high percentage of patients with elevated serum cystatin C (71\%), which indicates initial damage to the glomerulus.

Our results are consistent with other reports from South-Eastern Europe [5, 6, 32]. Chiţu-Tișu and colleagues observed a similar prevalence of reduced BMD (17\% osteoporosis and $48 \%$ osteopenia) in 60 HIV-infected patients in Romania. They noted that patients with more prolonged exposure to non-nucleoside reverse transcriptase inhibitors were at higher risk of low BMD [6]. Another group noted a $47 \%$ prevalence of low lumbar BMD in Romanian patients, together with a relatively high prevalence of chronic kidney disease. In this study, there was a significant correlation between bone-related comorbidities and lower nadir CD4 cell counts [32].

Although some studies show greater bone loss in patients receiving $\mathrm{PI}$ regimens [9], other studies do not confirm this finding [27]. Administration of TDF was associated with a sharp decrease in BMD and several studies have shown that there is greater bone loss in patients with long-term viral suppression treated with TDF-containing regimens, compared with other regimens $[14,36]$. Two prospective studies have shown that patients with an initial highly active antiretroviral therapy (HAART) regimen containing TDF, have a significantly greater reduction in $B M D$ in the hip and spine, than patients with $\mathrm{ABC}$-containing regimens [13]. In addition, the ACTG5224 study demonstrated that regimens containing the protease inhibitors atazanavir-ritonavir $(A T Z / r)$ resulted in a significant decrease in BMD in the spine but not in the hip, compared with regimens containing EFV [24].

The mechanisms by which ART regimens lead to loss of BMD are not fully understood. However, it is thought that TDF affects bone density indirectly through proximal tubular toxicity, resulting in a loss of phosphate and increased bone turnover [15, 36], whereas EFV and PIs are believed to affect BMD through effects on the metabolism of vitamin $D[35$, 38]. Other antiretroviral drugs (dolutegravir, cobicistat and atazanavir) may also be nephrotoxic and have indirect effects on bone density [27, 38].

In conclusion, our results show that nearly a third of patients treated with ART have osteopenia or osteoporosis. The risk of low BMD was correlated with age and the duration of therapy but not with the therapeutic regimen. Based on our observations and the results of other studies, we recommend that BMD is measured regularly in HIV-positive patients and that calcium and vitamin D supplements are prescribed, under the supervision of specialists.

We intend to continue our research on BMD in HIVinfected patients by including more patients, as well as correlating BMD with the degree of immune suppression and viral load.

\section{Acknowledgments and funding}

The measurement of cystatin C levels and the DEXA scans were carried out within the framework of a scientific research project of the Bulgarian Society of Infectious Diseases, sponsored by the pharmaceutical company Merck Sharp and Dohme. Editorial assistance in the preparation of this manuscript was provided by Christine Drewienkiewicz from SuccinctChoice Medical Communications (London, UK), with financial support from Gilead Sciences Europe Ltd. (Uxbridge, UK).

\section{Conflict of interest}

None of the authors have any competing interests to declare. 


\section{REFERENCES}

1. Ando M, Yanagisawa N. Epidemiology, clinical characteristics, and management of chronic kidney disease in human immunodeficiency virus-infected patients. World J Nephrol 2015;4(3):388-95

2. Borissova AM, Rashkov R, Boyanov M et al. Femoral neck bone mineral density and 10-year absolute fracture risk in a national representative sample of Bulgarian women aged 50 years and older. Arch Osteoporos 2011;6:189-95.

3. Bruera D, Luna N, David DO et al. Decreased bone mineral density in HIV-infected patients is independent of antiretroviral therapy. AIDS 2003;17(13):1917-23.

4. Cazanave C, Dupon M, Lavignolle-Aurillac V et al. Reduced bone mineral density in HIV-infected patients: prevalence and associated factors. AIDS 2008;22(3):395-402.

5. Chitu-Tisu CE, Barbu EC, Lazar M et al. Body composition in HIV-infected patients receiving highly active antiretroviral therapy. Acta Clin Belg 2017;72(1):55-62

6. Chitu-Tisu CE, Barbu EC, Lazar M et al. Low bone mineral density and associated risk factors in HIV-infected patients. Germs 2016;6(2):50-9.

7. Danjuma MI, Mohamad-Fadzillah NH, Khoo S. An investigation of the pattern of kidney injury in HIV-positive persons exposed to tenofovir disoproxil fumarate: an examination of a large population database (MHRA database). Int J STD AIDS 2014;25(4):273-9

8. Deya-Martinez A, Fortuny C, Soler-Palacin P et al. Cystatin C: a marker for inflammation and renal function among HIV-infected children and adolescents. Pediatr Infect Dis J 2016;35(2):196-200.

9. Duvivier C, Kolta S, Assoumou L, Ghosn J, Rozenberg S, Murphy RL, et al. Greater decrease in bone mineral density with protease inhibitor regimens compared with nonnucleoside reverse transcriptase inhibitor regimens in HIV-1 infected naive patients. AIDS 2009;23(7):817-24.

10. Ezinga M, Wetzels JF, Bosch ME et al. Long-term treatment with tenofovir: prevalence of kidney tubular dysfunction and its association with tenofovir plasma concentration. Antivir Ther 2014;19(8):765-71.

11. Gallant JE, Parish MA, Keruly JC, Moore RD. Changes in renal function associated with tenofovir disoproxil fumarate treatment, compared with nucleoside reverse-transcriptase inhibitor treatment. Clin Infect Dis 2005;40(8):1194-8.

12. Goh SSL, Lai PSM, Tan ATB, Ponnampalavanar S. Reduced bone mineral density in human immunodeficiency virus-infected individuals: a meta-analysis of its prevalence and risk factors. Osteoporos Int 2017. doi: 10.1007/s00198-017-4305-8.

13. Grant PM, Cotter AG. Tenofovir and bone health. Curr Opin HIV AIDS 2016;11(3):326-32.

14. Grigsby IF, Pham L, Mansky LM et al. Tenofovir-associated bone density loss. Ther Clin Risk Manag 2010;6:41-7.

15. Hall AM, Hendry BM, Nitsch D, Connolly JO. Tenofovir-associated kidney toxicity in HIV-infected patients: a review of the evidence. Am J Kidney Dis 2011;57(5):773-80.

16. Hileman CO, Eckard AR, McComsey GA. Bone loss in HIV: a contemporary review. Curr Opin Endocrinol Diabetes Obes 2015;22(6):446-51.

17. Hojs R, Bevc $S$, Ekart R et al. Serum cystatin C-based equation compared to serum creatinine-based equations for estimation of glomerular filtration rate in patients with chronic kidney disease. Clin Nephrol 2008;70(1):10-7.

18. Hudec SM, Camacho PM. Secondary causes of osteoporosis. Endocr Pract 2013;19(1):120-8.
19. International Society for Clinical Densitometry (ISCD). 2017 Available from: https://www.iscd.org/official-positions/officialpositions/. [Accessed 22 November 2017].

20. Joffe I, Epstein S. Osteoporosis associated with rheumatoid arthritis: pathogenesis and management. Semin Arthritis Rheum 1991;20(4):256-72.

21. Kirilov G. Laboratory diagnosis of parathyroid gland diseases and bone mineral metabolism. In: Paradigm, editor. Hormonal and Functional Diagnosis of Endocrine Diseases; 2012.

22. Kohlmeier L, Gasner C, Bachrach LK, Marcus R. The bone mineral status of patients with Marfan syndrome. J Bone Miner Res 1995;10(10):1550-5.

23. Lucas K, Behrens BA, Nolte I et al. Comparative investigation of bone mineral density using CT and DEXA in a canine femoral model. J Orthop Res 2017. doi: 10.1002/jor.23574.

24. McComsey GA, Kitch D, Daar ES et al. Bone mineral density and fractures in antiretroviral-naive persons randomized to receive abacavir-lamivudine or tenofovir disoproxil fumarateemtricitabine along with efavirenz or atazanavir-ritonavir: AIDS Clinical Trials Group A5224s, a substudy of ACTG A5202. J Infect Dis 2011;203(12):1791-801.

25. McComsey GA, Tebas P, Shane E et al. Bone disease in HIV infection: a practical review and recommendations for HIV care providers. Clin Infect Dis 2010;51(8):937-46.

26. Moran CA, Weitzmann MN, Ofotokun I. Bone loss in HIV infection. Curr Treat Options Infect Dis 2017;9(1):52-67.

27. Pan G, Yang Z, Ballinger SW, McDonald JM. Pathogenesis of osteopenia/osteoporosis induced by highly active anti-retroviral therapy for AIDS. Ann N Y Acad Sci 2006;1068:297-308.

28. Powderly WG. Osteoporosis and bone health in HIV. Curr HIVIAIDS Rep 2012;9(3):218-22.

29. Rosen CJ. The epidemiology and pathogenesis of osteoporosis. In: De Groot LJ, Chrousos G, Dungan K, Feingold KR Grossman A et al. Endotext. South Dartmouth (MA): MDText. com, Inc.; 2000.

30. Sandhu SK, Hampson G. The pathogenesis, diagnosis, investigation and management of osteoporosis. J Clin Pathol 2011;64(12):1042-50.

31. Shlipak MG, Mattes MD, Peralta CA. Update on cystatin C: incorporation into clinical practice. Am J Kidney Dis 2013;62(3):595-603.

32. Streinu-Cercel A, Sandulescu O, Ceapraga G, et al. Prevalence of osteo-renal impairment in the Romanian HIV cohort. BMC Infect Dis 2016;16 Suppl 1:93.

33. Van Dijk FS. Genetics of osteoporosis in children. Endocr Dev 2015;28:196-209.

34. Wasserman P, Rubin DS. Highly prevalent vitamin D deficiency and insufficiency in an urban cohort of HIVinfected men under care. AIDS Patient Care STDS 2010;24(4):223-7.

35. Welz T, Childs K, Ibrahim F et al. Efavirenz is associated with severe vitamin $\mathrm{D}$ deficiency and increased alkaline phosphatase. Aids 2010;24(12):1923-8.

36. Woodward CL, Hall AM, Williams IG et al. Tenofovir-associated renal and bone toxicity. HIV Med 2009;10(8):482-7.

37. World Health Organization. WHO scientific group on the assessment of osteoporosis at primary health care level 2007. Available from: http://www.who.int/chp/topics/Osteoporosis. pdf. [Accessed 22 November 2017].

38. Yancheva N. Complications of antiretroviral therapy in HIVinfected patients. Vitamin D deficiency. PhD Thesis.

39. Yancheva N, Nikolova M, Alexandrova M et al. Deficiency of vitamin $D$ in HIV infected patients and its effect on some of the immunological parameters. World J AIDS 2015;5(3):182-8.

40. Yancheva N, Tchervenyakova T, Gabarska I, Elenkov I. Deficiency of vitamin D in HIV infected patients and its effect on some of the biochemical parameters. Indian J Pharm Sci 2016;3(2):57-64 